\title{
Ferromagnetic quantum critical point in URhGe doped with Ru
}

\author{
N. T. Huy, ${ }^{1}$ A. Gasparini, ${ }^{1}$ J. C. P. Klaasse,${ }^{1}$ A. de Visser,,${ }^{1} *$ S. Sakarya, ${ }^{2}$ and N. H. van Dijk ${ }^{2}$ \\ ${ }^{1}$ Van der Waals-Zeeman Institute, University of Amsterdam, Valckenierstraat 65, 1018 XE Amsterdam, The Netherlands \\ ${ }^{2}$ Department of Radiation, Radionuclides \& Reactors, Delft University of Technology, Mekelweg 15, 2629 JB Delft, The Netherlands
}

(Received 13 March 2007; published 8 June 2007)

\begin{abstract}
We have investigated the thermal, transport, and magnetic properties of $\mathrm{URh}_{1-x} \mathrm{Ru}_{x} \mathrm{Ge}$ alloys near the critical concentration $x_{c r}=0.38$ for the suppression of ferromagnetic order. The Curie temperature vanishes linearly with $x$ and the ordered moment $m_{0}$ is suppressed in a continuous way. At $x_{c r}$, the specific heat varies as $c$ $\sim T \ln T$, the $\gamma$ value $c /\left.T\right|_{0.5 \mathrm{~K}}$ is maximum, and the temperature exponent of the resistivity $\rho \sim T^{n}$ attains a minimum value $n=1.2$. These observations provide evidence for a ferromagnetic quantum phase transition. Interestingly, the coefficient of thermal expansion and the Grüneisen parameter $\Gamma$ remain finite at $x_{c r}$ (down to $T=1 \mathrm{~K}$ ), which is at odds with recent scaling results for a metallic quantum critical point.
\end{abstract}

DOI: 10.1103/PhysRevB.75.212405

PACS number(s): 75.30.Mb, 71.10.Hf, 75.40.Cx

In recent years, interest has continued to grow in materials that exhibit a quantum phase transition (QPT), i.e., a transition at zero temperature driven by quantum fluctuations. ${ }^{1}$ QPTs are fundamentally different from their classical counterparts at finite $T$, where the transition is due to thermal fluctuations of the order parameter. QPTs can be induced in a wide range of materials, such as correlated metals, ${ }^{2}$ cuprate superconductors, ${ }^{3}$ common metals,${ }^{4}$ and the two-dimensional electron gas. ${ }^{5}$ This is accomplished by adjusting a control parameter (e.g., pressure $p$, doping $x$, magnetic field $B$, or electron density) in order to tune the system to a quantum critical point (QCP). At this point, the quantum critical fluctuations give rise to unusual temperature laws [non-Fermiliquid behavior (nFL)] for the magnetic, thermal, and transport parameters, ${ }^{6,7}$ and new collective states may emerge, e.g., unconventional superconducting ${ }^{8}$ or electronic states. ${ }^{9}$ This in turn calls for novel concepts and theories. ${ }^{2,10,11}$ In order to provide a fruitful testing ground, it is important to identify new systems and to investigate their critical behavior.

Strongly correlated electron systems, notably heavyfermion compounds based on the $f$ elements $\mathrm{Ce}, \mathrm{Yb}$, or $\mathrm{U}$, are especially suited to study magnetic-to-nonmagnetic QPTs, because the ordering temperatures are low $(\sim 10 \mathrm{~K})$ and the exchange interaction can be modified relatively easily by an external control parameter. Currently, there are two central questions that are being addressed by studying QPTs in these materials. The first issue is the fate of the quasiparticles when the antiferromagnetic (AF) or ferromagnetic (FM) phase is entered. In the conventional scenario, a spin density wave is formed ${ }^{6,7}$ and the quasiparticles preserve their itinerant character [as in $\mathrm{CeIn}_{3-x} \mathrm{Sn}_{x}$ (Ref. 12)]. Because the itinerant model is unable to account for the nFL behavior in certain materials, an alternative local quantum criticality model has been put forward..$^{2,10,11}$ Here, the quasiparticles (Kondo-screened moments) decompose at the critical point in conduction electrons and local $f$ moments that undergo magnetic order $\left[\right.$ as in $\mathrm{CeCu}_{6-x} \mathrm{Au}_{x}$ (Ref. 2) and $\mathrm{YbRh}_{2}\left(\mathrm{Si}_{1-x} \mathrm{Ge}_{x}\right)_{2}$ (Ref. 13)]. The second captivating issue is the emergence of unconventional superconducting (SC) states near the pressure induced QCPs in $\mathrm{CePd}_{2} \mathrm{Si}_{2}, \mathrm{CeIn}_{3},{ }^{8}$ and $\mathrm{UGe}_{2} \cdot{ }^{14}$ Evidence is at hand that in these materials un- conventional pairing is realized ( $d$-wave pairing for the AF and $p$-wave pairing for the FM systems). This strongly suggests Cooper pairing mediated by AF or FM spin fluctuations rather than by phonons. The coexistence of FM order and SC in $\mathrm{UGe}_{2}$ [and possibly in UIr (Ref. 15)] under pressure is uncommon in nature and attracts much attention.

In this Brief Report, we provide evidence for a ferromagnetic QPT in URhGe doped with Ru. Our research is motivated by the unique properties of the parent compound URhGe at ambient pressure: (i) SC below $T_{s}=0.25 \mathrm{~K}$ coexists with itinerant FM order (Curie temperature $\left.T_{C}=9.5 \mathrm{~K}\right)^{16}$ and (ii) reentrant $\mathrm{SC}$ is induced by applying a large magnetic field $(B \sim 12 \mathrm{~T}) .{ }^{17}$ These observations immediately prompted the question whether one can tune URhGe to a FM QCP by mechanical or chemical pressure, with the objective of probing the quantum critical fluctuations and possibly linking these to the SC pairing mechanism. Resistivity measurements under hydrostatic pressure, however, revealed that $T_{C}$ increases at a rate of $0.065 \mathrm{~K} / \mathrm{kbar}^{18}$ Also, upon the application of uniaxial pressure $T_{C}$ increases as was extracted from the Ehrenfest relation. ${ }^{19}$ As regards to chemical pressure, the best candidate dopants are $\mathrm{Ru}$ and $\mathrm{Co}$, since among the neighboring isostructural UTX compounds (T $=$ transition metal and $X=\mathrm{Ge}$ or $\mathrm{Si}$ ) only URuGe and UCoGe have a paramagnetic ground state. ${ }^{20,21}$ Indeed, FM order in URhGe can be suppressed by replacing $\mathrm{Rh}$ by $\mathrm{Ru}$ and vanishes at 38 at. \% Ru. ${ }^{22,23}$ Here, we investigate the thermal, transport, and magnetic properties of $\mathrm{URh}_{1-x} \mathrm{Ru}_{x} \mathrm{Ge}$ alloys near the critical concentration $x_{c r}=0.38$. The observed nFL $T$ dependencies of the specific heat and electrical resistivity, together with the smooth suppression of the ordered moment, provide evidence for a continuous FM QPT. This classifies $\mathrm{URh}_{1-x} \mathrm{Ru}_{x} \mathrm{Ge}$ as one of the scarce $f$-electron systems in which a FM QCP can be reached by doping (a FM QPT was also reported for $\mathrm{CePd}_{1-x} \mathrm{Rh}_{x},{ }^{24}$ but here the transition is "smeared").

Polycrystalline $\mathrm{URh}_{1-x} \mathrm{Ru}_{x} \mathrm{Ge}$ samples with $0.0 \leqslant x$ $\leqslant 0.60$ were prepared by arc-melting the constituents $\mathrm{U}, \mathrm{Rh}$, and $\mathrm{Ru}($ all $3 N)$ and $\mathrm{Ge}(5 N)$ under a high-purity argon atmosphere in a water-cooled copper crucible. The as-cast samples were wrapped in Ta foil and annealed under high vacuum in quartz tubes for 10 days at $875^{\circ} \mathrm{C}$. Samples were 


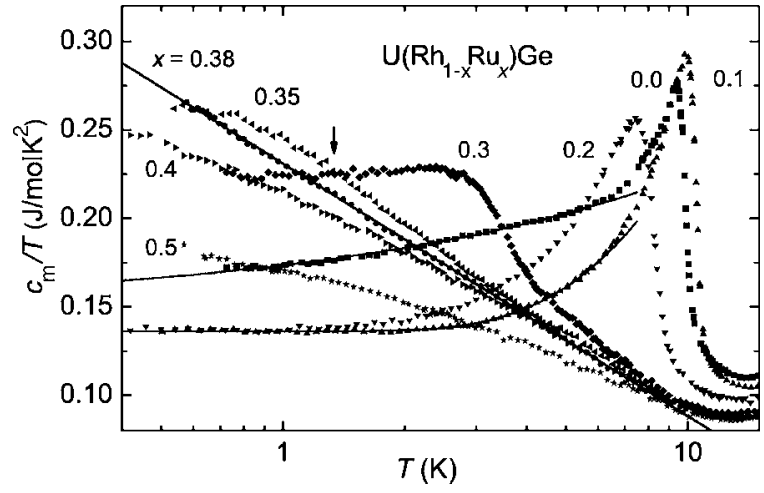

FIG. 1. $f$-electron specific heat of $\mathrm{URh}_{1-x} \mathrm{Ru}_{x} \mathrm{Ge}$ plotted as $c_{m} / T$ vs $\log T$ for $0 \leqslant x \leqslant 0.50$ as indicated. For $x \leqslant 0.10$, the data are fitted to $c_{m}(T)=\gamma T+\delta T^{3 / 2} e^{-\Delta / k_{B} T}$ with $\gamma, \delta$, and $\Delta / k_{B}$ values of $0.150, \quad 0.146$, and $0.136 \mathrm{~J} / \mathrm{mol} \mathrm{K}^{2}, \quad 0.024, \quad 0.041$, and $0.094 \mathrm{~J} / \mathrm{mol} \mathrm{K}^{5 / 2}$, and $0,6.5$, and $10.6 \mathrm{~K}$ for $x=0,0.05$, and 0.10 , respectively (solid lines for $x=0$ and $x=0.10$; data for $x=0.05$ not shown). The arrow indicates $T_{C}$ for $x=0.35$. For $x_{c r}=0.38, c_{m} / T$ $\sim \ln T$ over one and a half decade in $T$ (straight solid line).

cut by spark erosion. Electron probe microanalysis showed the single phase nature of the samples within the resolution of $2 \%$. X-ray powder diffraction confirmed the orthorhombic TiNiSi structure (space group Pnma). ${ }^{25,26}$ Upon substituting $\mathrm{Ru}$, the unit-cell volume $\Omega=224.3 \AA^{3}$ of URhGe decreases linearly at a rate of $0.067 \AA^{3} /$ at. \% Ru (i.e., $\Delta \Omega=1.1 \%$ at $x_{c r}$ ) in an anisotropic way, the main effect being the reduction of the $a$ lattice parameter. ${ }^{23}$

The specific heat $c(T)$ was measured down to $0.4 \mathrm{~K}$ using a semiadiabatic method in a homebuilt ${ }^{3} \mathrm{He}$ system. Electrical resistivity $\rho(T)$ data were collected in a commercial ${ }^{3} \mathrm{He}$ system (Heliox, Oxford Instruments, $T \geqslant 0.25 \mathrm{~K}$ ) using a low-frequency ac-resistance bridge. The thermal expansion $\alpha(T)$ was measured using a parallel-plate capacitance dilatometer in the $T$ range $1-15 \mathrm{~K}$. The dc magnetization $M(T)(T \geqslant 1.8 \mathrm{~K})$ was obtained using a Quantum Design superconducting quantum interference device magnetometer. Temperature scans in magnetic fields $B$ up to $5 \mathrm{~T}$ were made after field cooling.

The overall effect of Ru doping on ferromagnetism in $\mathrm{URhGe}$ is presented in Fig. 1, where we have plotted the $f$-electron specific heat $c_{m}$, obtained after subtracting the lattice contribution $\left[c_{\text {lat }}=\beta T^{3}\right.$ for $T \leqslant 20 \mathrm{~K}$ with $\beta=0.60$ $\times 10^{-3} \mathrm{~J} / \mathrm{mol} \mathrm{K}^{4}$ (Ref. 25)], as $c_{m} / T$ vs $\log T$ for $0 \leqslant x$ $\leqslant 0.50$. Upon doping, $T_{C}$ initially increases, but for $x$ $\geqslant 0.10$ the ordering peak shifts toward lower $T$ and weakens. Values of $T_{C}(x)$, identified by the inflection points in $c / T$ vs $T$ (on a linear $T$ scale) at the high $T$ side of the peaks, are traced in Fig. 2(a) and are in excellent agreement with the values determined from $M(T)$ and $\rho(T) .{ }^{22}$ For $x \geqslant 0.20, T_{C}$ decreases linearly with $x$ at a rate of $0.45 \mathrm{~K} /$ at. $\% \mathrm{Ru}$. For $x=0$, the magnetic specific heat for $T \leqslant 5 \mathrm{~K}$ is described by $c_{m}(T)=\gamma T+\delta T^{3 / 2}$, where $\gamma$ is the linear coefficient of the electronic specific heat and the second term is the spin-wave contribution. ${ }^{27}$ The values for $\gamma$ and $\delta$ extracted by fitting the data (see Fig. 1) are in good agreement with the values reported in Ref. 25. Upon doping $\mathrm{Ru}$, an energy gap $\Delta$ opens in

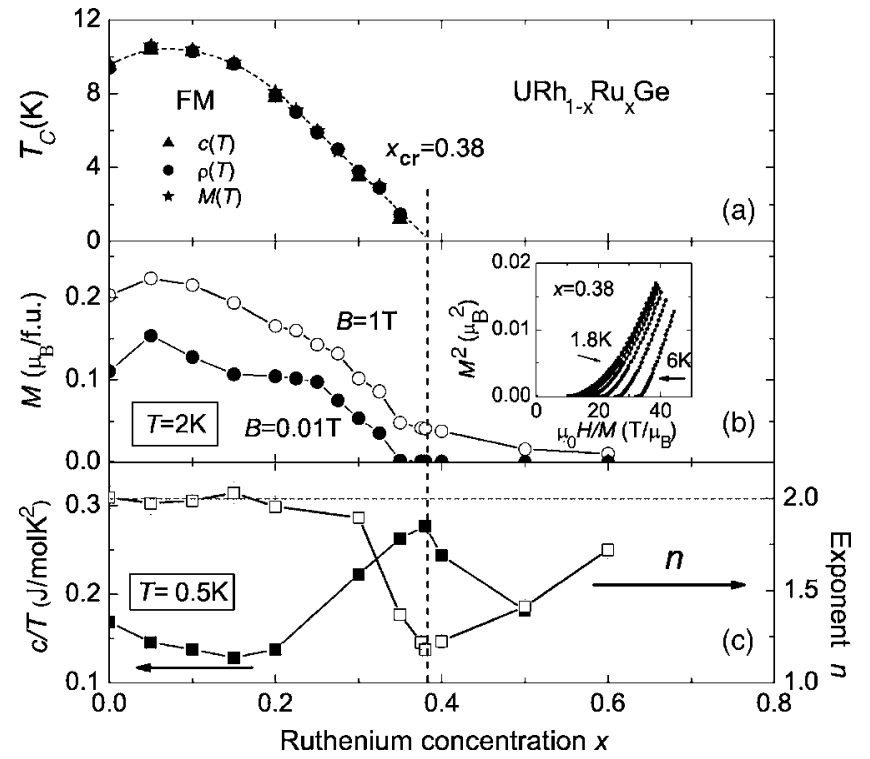

FIG. 2. (a) Curie temperature of $\mathrm{URh}_{1-x} \mathrm{Ru}_{x} \mathrm{Ge}$ determined from $c(T)(\mathbf{\Delta}), \rho(T)(\mathbf{\bullet})$, and $M(T)(\star)$. The critical Ru content is $x_{c r}$ $=0.38$ (vertical dashed line). (b) Magnetization $M$ at $2 \mathrm{~K}$ in $B$ $=0.01(\bigcirc)$ and $1 \mathrm{~T}(\bigcirc)$. Inset: Arrott plot for $x=0.38$ at $1.8 \mathrm{~K} \leqslant T$ $\leqslant 6 \mathrm{~K}$. (c) $c / T$ at $T=0.5 \mathrm{~K}(\boldsymbol{\square})$ and the exponent $n(\square)$ of $\rho \sim T^{n}$. The horizontal dashed line indicates $n=2$.

the magnon spectrum and the specific heat for $x=0.05$ and 0.10 now follows the relation $(T \leqslant 5 \mathrm{~K}) \quad c_{m}(T)=\gamma T$ $+\delta T^{3 / 2} e^{-\Delta / k_{B} T}$ (Ref. 27) (see fits in Fig. 1). The most important result of our specific-heat experiments, however, is the pronounced $c_{m}(T)=-b T \ln \left(T / T_{0}\right)$ dependence for $x_{c r}$, where $b=0.062 \mathrm{~J} / \mathrm{mol} \mathrm{K}^{2}$ and $T_{0}=41 \mathrm{~K}$. This $\mathrm{nFL}$ term is observed over one and a half decade in $T(0.5-9 \mathrm{~K})$. At $x_{c r}$ $c /\left.T\right|_{0.5 \mathrm{~K}}(x)$ has a maximum [Fig. 2(c)]. The total $f$-electron entropy obtained by integrating $c_{m} / T$ vs $T$ between 0.5 and $\sim 15 \mathrm{~K}$ amounts to $\sim 0.48 R \ln 2$ for $x=0$ and decreases to $0.33 R \ln 2$ at $x_{c r}$. Its small value confirms the itinerant nature of the FM transition [the ordered moment $m_{0}$ is $0.4 \mu_{B}$ for $x=0$ (Refs. 16 and 25)].

The electrical resistivity of $\mathrm{URh}_{1-x} \mathrm{Ru}_{x} \mathrm{Ge}(x \leqslant 0.60)$ at high $T$ (Ref. 23) shows the behavior typical for a FM Kondo lattice. The data for $x=0.38$ are shown in the inset in Fig. 3, where the maximum near $130 \mathrm{~K}$ signals the formation of the Kondo lattice. For the FM compounds at low $T$, a kink in $\rho(T)$ [and maximum in $d \rho(T) / d T$ ] marks $T_{C}$. For all doped samples, the total resistivity drop in the $T$ interval $0-300 \mathrm{~K}$ is $\sim 150-250 \mu \Omega \mathrm{cm}$, which is usual for uranium intermetallics. ${ }^{21}$ However, the residual resistivity values $\rho_{0}$ are large $(\sim 200-300 \mu \Omega \mathrm{cm})$, which is due to the brittleness of the samples (cracks). Consequently, the residual resistance ratio values $[R(300 \mathrm{~K}) / R(0 \mathrm{~K})]$ are small $(\sim 2)$. In Fig. 3, we show $\rho(T)$ at low $T$ for $0.10 \leqslant x \leqslant 0.60$. For a FM with gapped magnon modes $\rho(T)=\rho_{0}+A T^{n}$ $+B T \Delta e^{-\Delta / k_{B} T}\left(1+2 k_{B} T / \Delta\right),{ }^{28}$ where the second term is the electron-electron scattering term (i.e., the FL term when $n$ $=2$ ) and the third term yields the scattering from magnons. For $x=0.10$ and 0.20 , fits reveal that the second term is dominant $(A \gg B)$ and $\rho(T) \sim T^{2.0 \pm 0.1}$ over a wide $T$ range in the FM state (see Fig. 3). Therefore, we conclude that scat- 


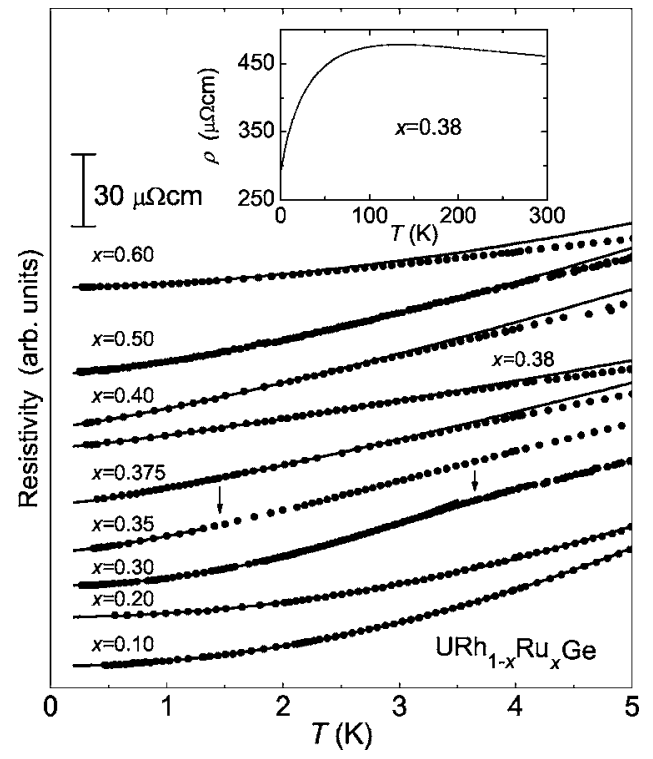

FIG. 3. Resistivity of $\mathrm{URh}_{1-x} \mathrm{Ru}_{x}$ Ge for $0.10 \leqslant x \leqslant 0.60$. The bar gives the absolute scale. The arrows for $x=0.30$ and 0.35 indicate $T_{C}$ obtained from additional data sets. The solid lines are fits to $\rho(T)=\rho_{0}+A T^{n}$. For $x \leqslant 0.3, n=2.0 \pm 0.1$. For $x_{c r}=0.38, n=1.2$ is minimum. Inset: Resistivity for $x=0.38$ up to $300 \mathrm{~K}$.

tering from magnons can be neglected in our polycrystalline samples and we restrict the analysis to fitting $\rho(T)=\rho_{0}$ $+A T^{n}$ (see Fig. 3). The values of $n$ extracted (by taking the best fit over the largest $T$ interval) are shown in Fig. 2(c). $n(x)$ attains a minimum value $n=1.2$ at $x_{c r}$, followed by a slow recovery to the FL value $n=2$ there above.

The magnetization $M(T)$ for all samples was measured in $B=0.01$ and $1 \mathrm{~T}$ down to $1.8 \mathrm{~K}$. In addition, $M(B)$ was measured at fixed $T$ in order to produce Arrott plots $\left(M^{2}\right.$ vs $B / M) .\left.\quad M\right|_{2 \mathrm{~K}}$ values are traced in Fig. 2(b). For pure URhGe, $\left.\quad M\right|_{2 \mathrm{~K}}$ in $1 \mathrm{~T} \simeq 0.2 \mu_{B}$ in agreement with the polycrystalline average $\frac{1}{2} m_{0}$ for a uniaxial FM $\left(m_{0}=0.4 \mu_{B}\right.$ directed along the $c$ axis $\left.{ }^{16}\right)$. In $0.01 \mathrm{~T}$, a reduced value $\left.M\right|_{2 \mathrm{~K}} \simeq 0.11 \mu_{B}$ is observed due to demagnetizing effects. Values of $T_{C}$ [Fig. 2(a)] were determined from the inflection points in $M(T)$ in $0.01 \mathrm{~T}$ and from the Arrott plots. For $x \geqslant 0.38$, the Arrott plots $(T \geqslant 1.8 \mathrm{~K})$ no longer indicate magnetic order [see inset in Fig. 2(b) for $x=0.38$ ]. The most important feature of the data is the gradual decrease of $\left.M\right|_{2 \mathrm{~K}}(x)$. For $B=0.01 \mathrm{~T},\left.M\right|_{2 \mathrm{~K}}(x)$ smoothly goes to 0 at $x=0.35\left(T_{C}=1.3 \pm 0.1 \mathrm{~K}\right)$, while for $B=1 \mathrm{~T}$ a finite field induced $\left.M\right|_{2 \mathrm{~K}}$ remains. We conclude that the FMparamagnetic transition as a function of $x$ is a continuous (second order) phase transition.

In Fig. 4, we show the coefficient of volume thermal expansion $\beta(T)$ for $x_{c r}=0.38$ at $T \geqslant 1 \mathrm{~K}$. The data (solid line) are obtained by averaging $\alpha_{i}(T)$ measured for three orthogonal directions on the polycrystalline sample $\left(\beta=\Sigma_{i} \alpha_{i}\right)$ in order to eliminate possible anisotropy effects due to crystallites with preferred orientations. The $T$ dependence of $\beta$ at low $T$ is weaker than that of the specific heat (see Fig. 4). Concurrently, the Grüneisen ratio $\Gamma=V_{m} \beta / \kappa c$ decreases below $T$ $\sim 7 \mathrm{~K}$ [here the molar volume $V_{m}=3.36 \times 10^{-5} \mathrm{~m}^{3} / \mathrm{mol}$ and

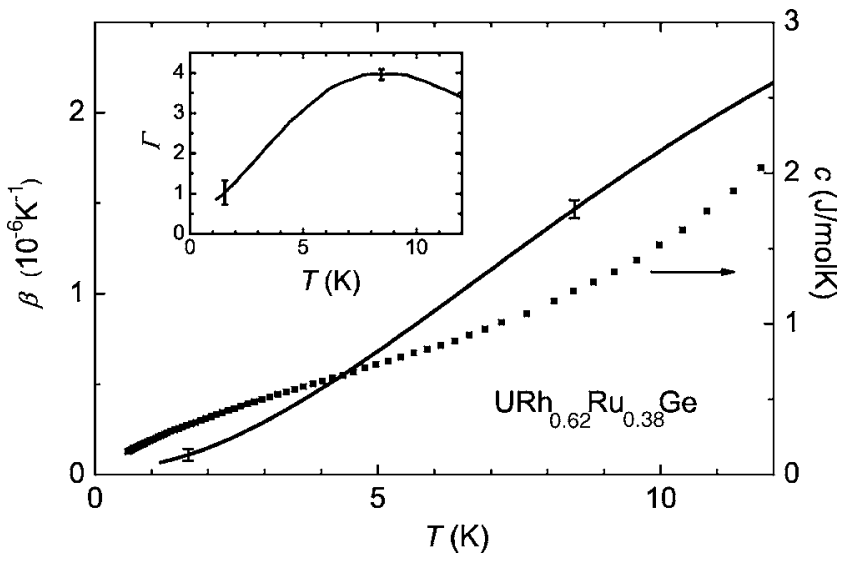

FIG. 4. Coefficient of volume thermal expansion $\beta(T)$ (solid line) and specific heat $c(T)(\bullet)$ of $\mathrm{URh}_{0.62} \mathrm{Ru}_{0.38} \mathrm{Ge}$. Inset: Grüneisen ratio $\Gamma$ as a function of $T$.

isothermal compressibility $\kappa \simeq 10^{-11} \mathrm{~Pa}^{-1}$ (Ref. 23)]. The quasilinear behavior of $\Gamma(T)$ for $1 \mathrm{~K} \leqslant T \leqslant 5 \mathrm{~K}$ suggests an unusual $T$ variation of $\beta$, i.e., roughly proportional to $T^{2} \ln T$.

Having documented the critical behavior of the $\mathrm{URh}_{1-x} \mathrm{Ru}_{x} \mathrm{Ge}$ alloys, we conclude that our $c(T), \rho(T)$, and $M(T)$ data provide evidence for a continuous FM QPT with $x_{c r}=0.38$. The most compelling evidence is the specific heat $c_{c r} \sim T \ln \left(T / T_{0}\right)$ observed over one and a half decade in $T$ (Fig. 1) ${ }^{7}$ and the concomitant maximum in $c /\left.T\right|_{0.5 \mathrm{~K}}(x)$ [Fig. 2(c)]. The temperature $T_{0}=41 \mathrm{~K}$ is large, which indicates that our $c(T)$ experiments down to $T=0.4 \mathrm{~K}\left(T / T_{0} \simeq 0.01\right)$ indeed probe the quantum critical regime. It will be interesting to investigate whether the $c / T \sim \ln T$ behavior persists even at lower $T$. Eventually, however, $c / T$ will saturate because of crystallographic disorder inherent to the $\mathrm{URh}_{1-x} \mathrm{Ru}_{x} \mathrm{Ge}$ alloys. Further support for a QCP is provided by the critical behavior in the resistivity $\rho_{c r} \sim T^{1.2}$ up to $2 \mathrm{~K}$. The exponent $n(x)$ has a pronounced minimum at $x_{c r}$ [Fig. 2 (c)]. The value $n=1.2$ is smaller than the value $n=5 / 3$ predicted for a clean FM QCP. ${ }^{29}$ This is not unexpected as disorder reduces $n .^{30}$ The itinerant nature of the FM state and the smooth suppression of $m_{0}$ pointing to a continuous phase transition strongly suggest that the QPT in $\mathrm{URh}_{1-x} \mathrm{Ru}_{x} \mathrm{Ge}$ is of the Hertz-Millis type, ${ }^{6,7}$ albeit with modified exponents due to the effects of doping (notably emptying the $d$ band and alloy disorder). For instance, for an itinerant clean FM QPT, one expects $T_{C} \sim\left(x_{c}-x\right)^{3 / 4}$ (dimension $d=3$, dynamical critical exponent $z=3$ ), while we obtain $T_{C} \sim\left(x_{c}-x\right)$ over a wide range $0.20 \leqslant x \leqslant 0.35$. Deviations from the clean behavior are also observed in $f$-electron materials with a pressure induced continuous FM QPT, such as $\mathrm{CeSi}_{1.81} \cdot{ }^{31}$ On the other hand, for $d$-electron alloys with a continuous FM QPT [e.g., $\mathrm{Ni}_{x} \mathrm{Pd}_{1-x}$ (Ref. 32) and $\mathrm{Zr}_{1-x} \mathrm{Nb}_{x} \mathrm{Zn}_{2}$ (Ref. 33)], the data are to a large extent in agreement with the itinerant model. Further theoretical work is required to clarify these issues.

Finally, we discuss our results for the thermal expansion and the Grüneisen parameter. The finite $\Gamma$ value at low $T$ is at variance with the recent prediction of a diverging Grüneisen ratio $\Gamma \sim T^{-1 / z \nu}$ at the QCP ( $\nu$ is the correlation length exponent). ${ }^{34}$ For the case of an itinerant FM QCP, the scaling results are $\beta_{c r} \sim T^{1 / 3}$ and $c_{c r} \sim T \ln (1 / T)$, whence $\Gamma_{c r}$ 
$\sim \beta_{c r} / c_{c r} \sim\left[\left(T^{2 / 3} \ln (1 / T)\right]^{-1} .{ }^{34}\right.$ While the specific heat follows the expected behavior, the thermal expansion clearly does not $\left(\beta \sim T^{2} \ln T\right.$ for $\left.1 \mathrm{~K} \leqslant T \leqslant 5 \mathrm{~K}\right)$. With the value $T_{0}$ $=41 \mathrm{~K}$ extracted from $c_{c r}$, we calculate that $\Gamma_{c r}$ within the scenario of Ref. 34 should have a minimum near $8 \mathrm{~K}$ and diverge at lower $T$. This is obviously not the case experimentally (Fig. 4). The only other system for which the Grüneisen ratio near a FM QPT has been investigated so far is $\mathrm{CePd}_{1-x} \mathrm{Rh}_{x} \cdot{ }^{35}$ In this system, a nondiverging ( $T$-independent) $\Gamma$ was also observed in the critical regime.

In conclusion, we have investigated the thermal, transport, and magnetic properties of $\mathrm{URh}_{1-x} \mathrm{Ru}_{x} \mathrm{Ge}$ near the critical concentration for the suppression of FM order. At $x_{c r}=0.38$ $c \sim T \ln T$, the $\gamma$ value $c /\left.T\right|_{0.5 \mathrm{~K}}$ has a maximum and the $T$ exponent in the resistivity attains the nFL value $n=1.2$. Together with the gradual suppression of the ordered moment $m_{0}$, the data provide evidence for a continuous FM quantum phase transition. This offers the sole opportunity thus far to investigate FM spin fluctuations in URhGe under quantum critical conditions. The identification of the FM QCP at ambient pressure in URhGe doped with Ru paves the road to a host of experiments on this unique material.

This work was part of the research program of FOM (Dutch Foundation for Fundamental Research of Matter) and COST Action P16 ECOM.
*Electronic address: devisser@science.uva.nl

${ }^{1}$ See, e.g., S. Sachdev, Quantum Phase Transitions (Cambridge University Press, Cambridge, 1999).

${ }^{2}$ A. Schröder, G. Aeppli, R. Coldea, M. Adams, O. Stockert, H. v. Löhneysen, E. Bucher, R. Ramazashvili, and P. Coleman, Nature (London) 407, 351 (2000).

${ }^{3}$ D. van der Marel, H. J. A. Molegraaf, J. Zaanen, Z. Nussinov, F. Carbone, A. Damascelli, H. Eisaki, M. Greven, P. H. Kes, and M. Li, Nature (London) 425, 271 (2003).

${ }^{4}$ A. Yeh, Yeong-Ah Soh, J. Brooke, G. Aeppli, T. F. Rosenbaum, and S. M. Hayden, Nature (London) 419, 459 (2002).

${ }^{5}$ S. L. Sondhi, S. M. Girvin, J. P. Carini, and D. Shahar, Rev. Mod. Phys. 69, 315 (1997).

${ }^{6}$ J. Hertz, Phys. Rev. B 14, 1165 (1976).

${ }^{7}$ A. J. Millis, Phys. Rev. B 48, 7183 (1993).

${ }^{8}$ N. D. Mathur, F. M. Grosche, S. R. Julian, I. R. Walker, D. M. Freye, R. K. W. Haselwimmer, and G. G. Lonzarich, Nature (London) 394, 39 (1998).

${ }^{9}$ S. A. Grigera, P. Gegenwart, R. A. Borzi, F. Weickert, A. J. Schofield, R. S. Perry, T. Tayama, T. Sakakibara, Y. Maeno, A. G. Green, and A. P. Mackenzie, Science 306, 1154 (2004).

${ }^{10}$ Q. Si, S. Rabello, K. Ingersent, and J. L. Smith, Nature (London) 413, 804 (2001).

${ }^{11}$ P. Coleman, C. Pépin, Q. Si, and R. Ramazashvili, J. Phys.: Condens. Matter 13, R723 (2001).

${ }^{12}$ R. Küchler, P. Gegenwart, J. Custers, O. Stockert, N. CarocaCanales, C. Geibel, J. G. Sereni, and F. Steglich, Phys. Rev. Lett. 96, 256403 (2006).

${ }^{13}$ J. Custers, P. Gegenwart, H. Wilhelm, K. Neumaier, Y. Tokiwa, O. Trovarelli, C. Geibel, F. Steglich, C. Pépin, and P. Coleman, Nature (London) 424, 524 (2003).

${ }^{14}$ S. S. Saxena, P. Agarwal, K. Ahilan, F. M. Grosche, R. K. W. Haselwimmer, M. J. Steiner, E. Pugh, I. R. Walker, S. R. Julian, P. Monthoux, G. G. Lonzarich, A. Huxley, I. Sheikin, D. Braithwaite, and J. Flouquet, Nature (London) 406, 587 (2000).

${ }^{15}$ T. Akazawa, H. Hidaka, T. Fujiwara, T. C. Kobayashi, E. Yamamoto, Y. Haga, R. Settai, and Y. Ōnuki, J. Phys.: Condens. Matter 16, L29 (2004).

${ }^{16}$ D. Aoki, A. Huxley, E. Ressouche, D. Braithwaite, J. Flouquet, J. P. Brison, E. Lhotel, and C. Paulsen, Nature (London) 413, 613 (2001).
${ }^{17}$ F. Lévy, I. Sheikun, B. Grenier, and A. D. Huxley, Science 309, 1343 (2005).

${ }^{18}$ F. Hardy, A. Huxley, J. Flouquet, B. Salce, G. Knebel, D. Braithwaite, D. Aoki, M. Uhlarz, and C. Pfleiderer, Physica B 359361, 1111 (2005).

${ }^{19}$ S. Sakarya, N. H. van Dijk, A. de Visser, and E. Brück, Phys. Rev. B 67, 144407 (2003).

${ }^{20}$ R. Troć and V. H. Tran, J. Magn. Magn. Mater. 73, 389 (1988).

${ }^{21}$ V. Sechovský and L. Havela, in Handbook of Magnetic Materials, edited by K. H. J. Buschow (North-Holland, Amsterdam, 1998), Vol. 11, pp. 1-289.

${ }^{22}$ S. Sakarya, N. H. van Dijk, N. T. Huy, and A. de Visser, Physica B 378-380, 970 (2006).

${ }^{23}$ S. Sakarya, N. T. Huy, N. H. van Dijk, A. de Visser, M. Wagemaker, A. C. Moleman, T. J. Gortenmulder, J. C. P. Klaasse, M. Uhlarz, and H. v. Löhneysen, arXiv:cond-mat/0609557, J. Alloys Compd. (to be published).

${ }^{24}$ J. G. Sereni, R. Küchler, and C. Geibel, Physica B 359-361, 41 (2005).

${ }^{25}$ K. Prokeš, T. Tahara, Y. Echizen, T. Takabatake, T. Fujita, I. H. Hagmusa, J. C. P. Klaasse, E. Brück, F. R. de Boer, M. Diviš, and V. Sechovský, Physica B 311, 220 (2002).

${ }^{26}$ B. Lloret, Ph.D. thesis, University of Bordeaux I, 1988.

${ }^{27}$ See, e.g., A. Tari, The Specific Heat of Matter at Low Temperatures (Imperial College Press, London, 2003).

${ }^{28}$ N. Hessel Andersen and H. Smith, Phys. Rev. B 19, 384 (1979).

${ }^{29}$ T. Moriya, Spin Fluctuations in Itinerant Electron Magnets (Springer-Verlag, Berlin, 1985).

${ }^{30}$ See, e.g., C. Pfleiderer, S. R. Julian, and G. G. Lonzarich, Nature (London) 414, 427 (2001).

${ }^{31}$ S. Drotziger, C. Pfleiderer, M. Uhlarz, H. v. Löhneysen, D. Souptel, W. Löser, and G. Behr, Phys. Rev. B 73, 214413 (2006).

${ }^{32}$ M. Nicklas, M. Brando, G. Knebel, F. Mayr, W. Trinkl, and A. Loidl, Phys. Rev. Lett. 82, 4268 (1999).

${ }^{33}$ D. A. Sokolov, M. C. Aronson, W. Gannon, and Z. Fisk, Phys. Rev. Lett. 96, 116404 (2006).

${ }^{34}$ L. Zhu, M. Garst, A. Rosch, and Q. Si, Phys. Rev. Lett. 91, 066404 (2003).

${ }^{35}$ J. G. Sereni, T. Westerkamp, R. Küchler, N. Caroca-Canales, P. Gegenwart, and C. Geibel, Phys. Rev. B 75, 024432 (2007). 